\title{
Dinâmica temporal do estrato herbáceo-arbustivo de uma área de campo limpo úmido em Alto Paraíso de Goiás, Brasil
}

\author{
Chesterton Ulysses Orlando Eugênio ${ }^{1,4}$, Cássia Beatriz Rodrigues Munhoz ${ }^{2}$ e Jeanine Maria Felfili ${ }^{3 \dagger}$
}

Recebido em 26/09/2010. Aceito em 20/05/2011

\begin{abstract}
RESUMO
(Dinâmica temporal do estrato herbáceo-arbustivo de uma área de campo limpo úmido em Alto Paraíso de Goiás, Brasil). Este trabalho avaliou a dinâmica estrutural e florística de uma comunidade de espécies herbáceo-arbustivas de uma área de campo limpo úmido em Alto Paraíso de Goiás, o primeiro inventário realizado em 2000 (T0) e o segundo em 2007 (T1). A diversidade de Shannon entre os períodos foi comparada pelo teste- $t$ de Hutcheson e a similaridade florística, pelo índice de similaridade de Chao-Sørensen. As relações florísticas e a cobertura, entre os períodos e as linhas, foram avaliadas por meio de análises de correspondência retificada (DCA). Foram amostradas 98 espécies, 88 no T0 e 67 no T1, sendo 31 exclusivas do T0 e 10 do T1. A diversidade florística na comunidade foi elevada nos dois períodos, porém diferente entre esses $(t=7,12 ; p<0,001)$, devido a variação no número $\mathrm{e}$ cobertura das espécies. A similaridade entre os dois inventários foi alta (Chao-Sørensen $\pm \mathrm{IC}=0,841 \pm 0,074)$. A ordenação por DCA indicou relações entre a composição florística e a cobertura com o gradiente de umidade e de matéria orgânica no solo identificados em T0. Houve modificações nas linhas em zonas sazonais, as quais se tornaram mais semelhantes às linhas constantemente saturadas por água. Em um intervalo de sete anos o campo limpo úmido apresentou mudanças na composição florística e, principalmente na estrutura devido o aumento da cobertura de espécies perenes, cespitosas e entouceiradas, que foram favorecidas pela maior umidade no solo em resposta à elevação da pluviosidade da região.
\end{abstract}

Palavras-chave: Avaliação temporal, Chapada dos Veadeiros, diversidade, interseção na linha, Paspalum lineare

\begin{abstract}
(Temporal dynamics of the shrub and herbaceous layer of an area of moist grassland in Alto Paraíso de Goiás, Brazil). This study evaluated the floristic and structural dynamics of a community of herbaceous-shrub species in an area of moist grassland in Alto Paraíso de Goiás. The first inventory was undertaken in 2000 (T0) and the second in 2007 (T1). Shannon's diversity between the periods was compared by Hutchesons's $t$-test, and the floristic similarity by the Chao-Sørensen similarity index. Floristic composition and cover, between periods and lines, were evaluated by detrended correspondence analysis (DCA). We sampled 98 species, 88 at T0 and 67 at T1; 31 were unique to T0 and 10 to T1. Floristic diversity in the community was high in both periods, but different between them $(t=7.12, p<0.001)$, due to variation in species number and coverage. Similarity between the two surveys was high (Chao-Sørensen CI $= \pm 0.841 \pm 0.074$ ). The DCA ordination indicated relationships between the floristic composition and cover with a gradient of moisture and organic matter in the soil identified in T0. There were changes in the lines in the seasonal zones, which became more similar in those constantly saturated with water. During an interval of seven years the moist grassland showed changes in floristic composition and mainly in structure due to increased cover of the clumped tussock perennial species, which were favored by higher soil moisture due to high rainfall in the region.
\end{abstract}

Key words: Chapada dos Veadeiros, diversity, line intercept, Paspalum lineare, temporal evaluation

\footnotetext{
1 Universidade de Brasília, Departamento de Engenharia Florestal, Pós-Graduação em Ciências Florestais, Brasília, DF, Brasil

2 Universidade de Brasília, Departamento de Botânica, Brasília, DF, Brasil

3 Universidade de Brasília (UnB), Departamento de Engenharia Florestal, Brasília, DF, Brasil. In memoriam

4 Autor para correspondência: chesterton@ig.com.br
} 


\section{Introdução}

Interesses em pesquisas ecológicas de longa duração estão expandindo rapidamente, refletindo o crescente reconhecimento da importância em avaliar e resolver assuntos ambientais complexos, que incluam descrições da dinâmica da vegetação, assim como estudos experimentais de fatores mantenedores da diversidade de espécies (Bakker et al. 1996).

A relevância de se incluir nessas pesquisas comunidades úmidas deve-se à presença dessas em diferentes regiões no mundo (Junk 2003), e de sua importância na manutenção dos processos hidrogeológicos. O dinamismo e a heterogeneidade de paisagens com alagamentos temporários e perenes assumem funções importantes na filtragem e retenção da água, e na biodiversidade de plantas e animais típicos a esses ambientes (Junk 2003). A flutuação do excedente hídrico superficial no solo pode atuar como um filtro ecológico (Chase 2003), restringindo o número de espécies e selecionando as espécies que podem persistir dentro da comunidade tolerando as condições abióticas (Weiher \& Keddy 1995).

O campo limpo úmido é uma fitofisionomia campestre do Cerrado composta em sua ampla extensão por espécies herbáceo-subarbustivas, com representantes característicos de ambientes com flutuação de excedente hídrico subsuperficial (Tannus \& Assis 2004; Munhoz \& Felfili 2007), em que o componente lenhoso corresponde a menos de $5 \%$ da cobertura das espécies (Ribeiro \& Walter 2008). Ocorrem muitas vezes próximos às nascentes, em áreas com lençol freático superficial e entre as matas de galeria e o cerrado sensu stricto, sendo frequentemente uma área de transição entre essas fitofisionomias (Tannus \& Assis 2004; Munhoz \& Felfili 2006a; 2007; Ribeiro \& Walter 2008). Geralmente são encontrados sobre solos hidromórficos, glei ou orgânicos turfosos, em gradientes de umidade, desde permanente a sazonalmente inundáveis, em função das estações chuvosa e seca e das características de drenagem das áreas onde se encontram (Ribeiro \& Walter 2008).

A dinâmica de comunidades de plantas tem sido estudada por meio de pesquisas que avaliam as variações na estrutura e na composição florística ao longo do tempo e do espaço por meio de análises da composição, diversidade, produtividade, estratégia de vida, sucessão e estabilidade das espécies (Herben et al. 2000; Rees et al. 2001). Estudos sobre a estrutura e a dinâmica de campo limpo úmido e suas variáveis ambientais são essenciais para o delineamento de estratégias para a conservação da diversidade biológica, assim como para seu uso sustentável (Tannus \& Assis 2004; Munhoz \& Felfili 2006a; b). Tais estudos oferecem oportunidade de compreensão da dinâmica da vegetação relacionada às variações ambientais que podem ser usadas para inferir futuras respostas da vegetação em relação às mudanças climáticas globais (Parmesan \& Yohe 2003; Matesanz et al. 2009).
A variação na riqueza de espécies em uma mesma comunidade devido a diferenças locais nos níveis de flutuação do excedente hídrico no solo é comum, quer seja em áreas campestres na Venezuela (Sarmiento 1983; Sarmiento et al. 2004), como no Brasil (Cianciaruso \& Batalha 2008; Munhoz \& Felfili 2008; Munhoz et al. 2008), como também em vereda (Araújo et al. 2002). Pois os filtros ecológicos sazonais são severos, exigindo dos organismos distintas estratégias para sobreviverem e se desenvolverem aos diferentes regimes (Fukami et al. 2005). Em campo limpo úmido as flutuações na abundância e frequência das espécies foram, temporalmente, relacionadas à sazonalidade climática e, espacialmente as variações no excedente hídrico do solo (Munhoz 2003; Munhoz \& Felfili 2008; Cianciaruso \& Batalha 2008). Redução na riqueza em áreas campestres, ao longo dos anos, foi associada ao aumento nas populações de poucas espécies que se tornam mais abundantes (Chapin et al. 1995; Dunnett et al. 1998). Com o aumento da cobertura principalmente de subarbustos e gramíneas perenes, muitas vezes favorecidos pela elevação da pluviosidade após períodos de estiagens (Anderson \& Inouye 2001) ou por alterações experimentais que induziram o aumento da entrada de água no sistema, simulando a elevação de pluviosidade (Morecroft et al. 2004; Matesanz et al. 2009)

Tendo em vista a importância da comunidade herbáceosubarbustiva e a escassez de estudos de longa duração, este trabalho avaliou a dinâmica estrutural e florística da comunidade herbáceo-subarbustivas de uma área de campo limpo úmido na Fazenda Água Fria, Alto Paraíso de Goiás, após um intervalo de sete anos do primeiro inventário na área. O objetivo foi responder às seguintes questões: (i) houve diferença significativa na diversidade entre os dois períodos de amostragem?; (ii) qual é a similaridade florística entre os períodos amostrados? (iii) as linhas de amostragem se assemelham, floristicamente e estruturalmente, mais em relação a sua distribuição na área ou em função do tempo?; e (iv) a separação entre as linhas de amostragem sugeridas por Munhoz (2003) se manteve após sete anos?

\section{Material e métodos}

\section{Área de estudo}

O estudo foi realizado em um campo limpo úmido, próximo ao córrego Água Fria (1404'8,83” S e 47³0’33,1” W), situado na Fazenda Água Fria (FAF), localizada a cerca de $11 \mathrm{~km}$ à direita da rodovia de Alto Paraíso em sentido à Teresina de Goiás, na Chapada dos Veadeiros, a $1.482 \mathrm{~m}$ de altitude. A FAF é uma propriedade privada que explora o ecoturismo, sendo o campo limpo úmido uma área livre de ação antrópica, de pastejo e sem registro de incêndios desde 1995.

A Chapada dos Veadeiros apresenta campos limpos úmidos e uma alta variedade de outras fitofisionomias do Cerrado situadas em relevos suaves a fortemente ondula- 
dos, com predomínio de vegetação campestre e savânica nas áreas mais elevadas e florestal nos vales (Munhoz \& Proença 1998; Felfili 2007). A região apresenta uma flora típica e com espécies endêmicas (Munhoz \& Proença 1998; Simon \& Proença 2000; Filgueiras 2002; Munhoz \& Felfili 2006a), e trata-se talvez do maior centro de biodiversidade do estado de Goiás (Harley 1995).

O clima da região é do tipo Aw, segundo a classificação de Köppen (1948). Caracteriza-se por duas estações bem definidas. O período chuvoso, de outubro a abril, é quente com mínima de $13^{\circ} \mathrm{C}$ e máxima de $30^{\circ} \mathrm{C}$. A estação seca e fria, de maio a setembro, apresenta temperatura mínima de 4 a $8^{\circ} \mathrm{C}$ e máxima de 22 a $35^{\circ} \mathrm{C}$. As temperaturas médias anuais em Alto Paraíso são de 24 a $26^{\circ} \mathrm{C}$ (Felfili 2007). A precipitação média anual, segundo o Sistema Nacional de Informações sobre Recursos Hídricos (SNIRH) dos últimos 39 anos foi de $1486 \mathrm{~mm}$ (Fig. 1).

O campo limpo úmido ocupa uma área de 21 ha. Em 2000 o campo úmido foi subdividido em quatro subáreas, com base nos tipos fitofisionômicos adjacentes ao mesmo: (1) borda de cerrado rupestre, (2) borda de mata de galeria, (3) borda de vereda e (4) região central do campo limpo úmido. Após a estratificação foram sorteadas e instaladas 15 linhas de amostragem permanentes de $10 \mathrm{~m}$ em toda área. Sendo quatro linhas na primeira subárea (L1, L4, L5 e L7), três na segunda (L8, L9 e L10), três na terceira (L11, L12 e L14) e cinco na quarta (L2, L3, L6, L13 e L15) (Fig. 2), de acordo com o tamanho do estrato definido. Cada linha, unidade amostral (UA), foi demarcada e subdividida com vergalhões de ferro em segmentos de $1 \mathrm{~m}$ que representaram as subunidades amostrais (SuAS) para a amostragem da vegetação.

Os solos encontrados em sua maior porção são do tipo plintossolos pétricos concressionário típico, originário da decomposição de quartzitos, com textura média muito arenosa e drenagem moderada. Próximo à mata de galeria, o solo é hidromórfico, glei húmico, com grande quantidade de matéria orgânica (Munhoz \& Felfili 2006a). Munhoz (2003) observou uma alta correlação entre a distribuição das espécies e das linhas de amostragem com as variáveis ambientais do solo, principalmente em função da porcentagem de umidade gravimétrica e de matéria orgânica no solo, as quais foram fortemente correlacionadas com a estrutura herbáceo-arbustiva da comunidade em 2000. As linhas de amostragem L8, L9, L10, L11, L12 e L14 ocorreram sobre solos permanentemente saturados de água (umidade gravimétrica entre 100 e $600 \%$ ) e com elevado teor de matéria orgânica (entre 130 e 260 g.dm ${ }^{-3}$ ); L2, L7 e L13 apresentaram sobre solos inundados somente no auge da estação úmida (umidade gravimétrica entre 53 e $76 \%$ ) e com elevado teor de matéria orgânica (entre 75 e 100 g.dm ${ }^{-3}$ ); L1, L3, L4, L5 e L6 apresentaram sobre solos com menores porcentagens de umidade gravimétrica (12 a 26\%) e de matéria orgânica (3 e 19 g.dm ${ }^{-3}$ ) (Munhoz 2003).

\section{Amostragem}

O primeiro inventário foi realizado na segunda quinzena de abril no ano 2000 (T0) e o segundo em 2007 (T1) na primeira quinzena de maio. A determinação da composição e cobertura linear das espécies foi feita através do método de inventário de interseção na linha (Canfield 1941, 1950). O método consiste em traçar transectos sobre a vegetação a ser amostrada e anotar a projeção de cada espécie sob os mesmos. O comprimento de linha interceptado por uma espécie, dividido pelo comprimento total das espécies inventariadas sob a linha, refere-se à proporção da área coberta por aquela espécie. Com o auxílio de uma vareta de $1 \mathrm{~m}$, demarcada com uma fita métrica, colocada sobre cada SuA, fez-se a visualização da projeção horizontal das espécies na linha, na qual eram considerados todos os indivíduos com hábito herbáceo, subarbustivo e arbustivo. A ocorrência e o comprimento ocupado (projeção) de cada espécie, inclusive dos locais com solo descoberto, foi anotada por SuA ao longo das linhas amostradas, perfazendo um total de 150 SuAs de $1 \mathrm{~m}$ inventariadas.

\section{Parâmetros fitossociológicos}

Os cálculos de cobertura e frequência, absolutas e relativas, foram realizados pelas fórmulas de Kent \& Coker (1992), adaptados por Munhoz \& Felfili (2006a) para o componente herbáceo-subarbustivo, como se segue:

- Frequência absoluta: $F A_{i}=\left(\frac{n_{i}}{n}\right) \times 100$
- Frequência relativa: $F R_{i}=\left(\frac{F A_{i}}{\sum_{i=1}^{n} F A_{i}}\right) \times 100$

- Cobertura Absoluta: $C A_{i}=\sum_{i=1}^{n} P_{i} A$

- Cobertura Relativa: $C R_{i}=\left(\frac{C A_{i}}{\sum_{i=1}^{n} C A_{i}}\right) \times 100$

Onde: $n_{i}$ é número de UAs onde a espécie ( $i$ ) ocorreu; o $n$ é número total de UAs; $\sum_{i=1}^{n} F A_{i}$ é o somatório da frequência absoluta de todas as espécies; $\sum_{i=1}^{n} P_{i} A$ é a soma da projeção da espécie (i) em todas as SuAs; $\sum_{i=1}^{n} C A_{i}$ é o somatório da cobertura de todas as espécies em todas as SuAs. 


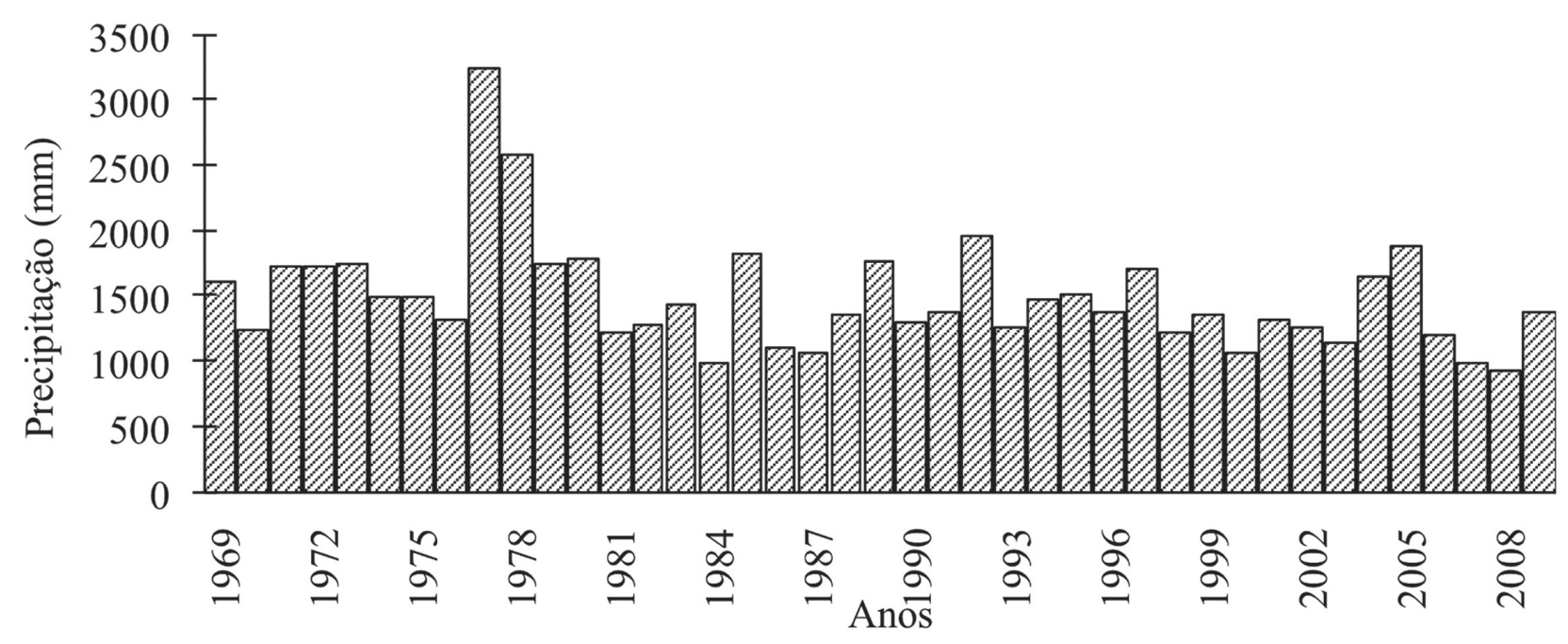

Figura 1. Valores médios anuais de precipitação de 1969 a 2009 em Alto Paraíso de Goiás, Goiás, Brasil. (Fonte: Banco de Dados Hidrometeorológicos - Módulo de Dados Qualitativo/Quantitativos do Sistema Nacional de Informações sobre Recursos Hídricos - SNIRH; Operadora da Estação: Furnas Centrais Elétricas S.A. http://www.ana.gov.br/portalsnirh. (Acesso em 25/02/2010).
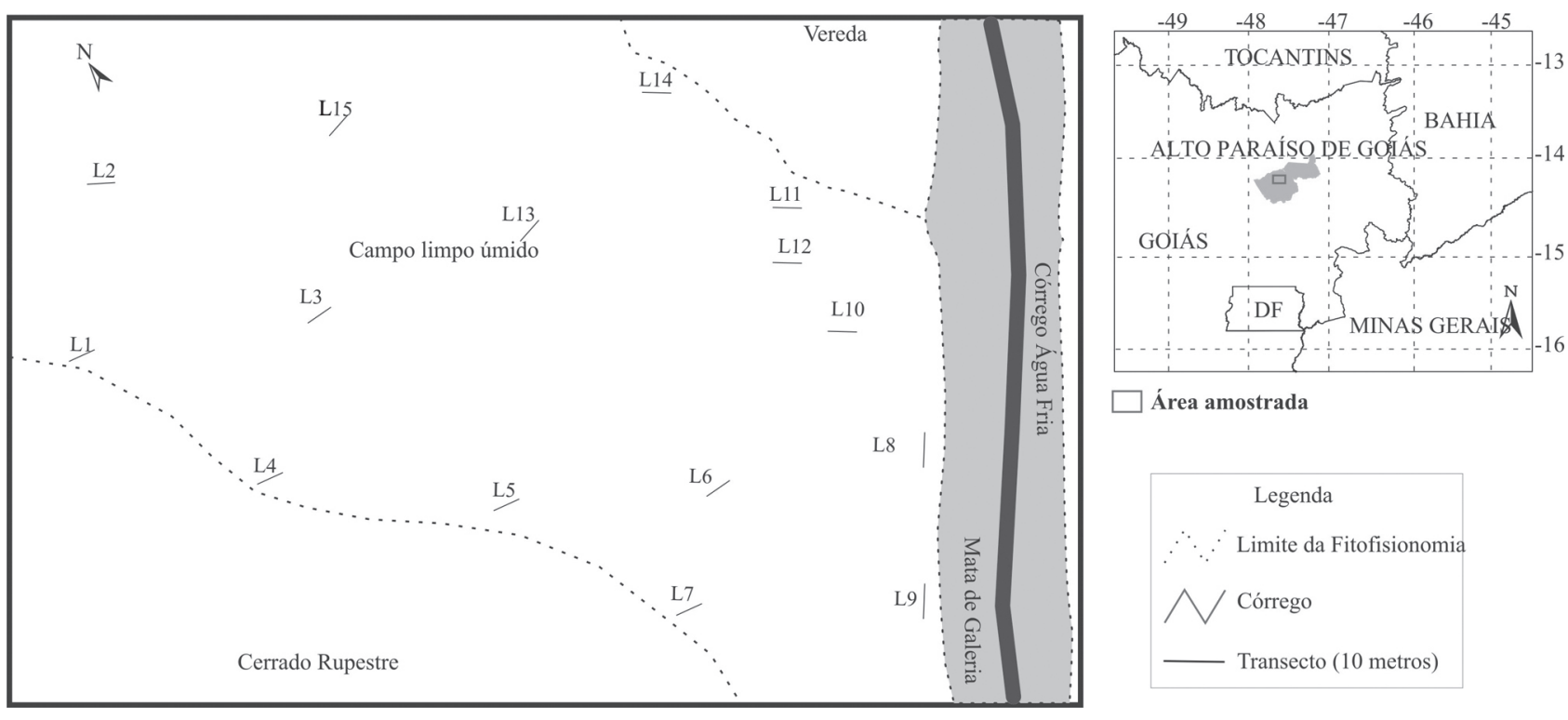

Figura 2. Esquema de distribuição das linhas de amostragem, em relação às fitofisionomias circunvizinhas, no campo limpo úmido na Fazenda Água Fria, Alto Paraíso de Goiás, Goiás, Brasil.

\section{Dinâmica da comunidade}

A cobertura e a frequência para todas as espécies amostradas na comunidade nos dois levantamentos foi comparada por meio do teste de Qui-quadrado (Zar 1999).

A diversidade florística, de cada período, foi calculada pelo índice de diversidade de Shannon na base $e\left(\mathrm{H}^{\prime}\right)$, adaptado por Munhoz \& Felfili (2006a), utilizando-se em vez do número de indivíduos, os valores de cobertura para espécie como uma medida de abundância, pois para o estrato herbáceo arbustivo a definição de indivíduo em campo torna-se dificultada pela presença de rebrotos ra- diculares de um mesmo indivíduo. A comparação quanto à significância entre os valores de H' nos dois períodos de inventário foi avaliada pelo teste- $t$ de Hutcheson $\alpha=0,05$ (Zar 1999), utilizando o software R versão 2.4 por meio do pacote Vegan (R Development Core Team 2006).

A similaridade entre os períodos amostrados foi avaliada pelo índice de similaridade de Chao-Sørensen (Chao et al. 2005; 2006). Utilizou-se uma matriz com o número atribuído a cada espécie encontrada (1...98), o número do inventário (T0 ou T1) e a cobertura dessa combinação, corrigindo, desta forma, os desvios de estimativa que podem ser produzidos em análises de similaridade baseadas 
somente na presença ou ausência (Chao et al. 2005; 2006). O índice Chao-Sørensen e o desvio padrão foram obtidos pelo programa EstimateS 8.0 (Colwell 2006). O intervalo de confiança do índice foi obtido pela multiplicação do desvio padrão pelo valor de $t_{0,05,2}$ para uma distribuição $t$ de Student com grau de liberdade $n-1$. A avaliação de que a similaridade seria maior entre os anos ou dentro dos anos foi verificada pela Análise de Variância de Similaridade (ANOSIM) que consiste na diferença entre os valores médios dos índices de similaridade das espécies entre as amostras $\left(r_{B}\right)$ e dentro de amostras $\left(r_{w}\right)$, por meio de um teste estatístico não-paramétrico. $O$ resultado pode variar de $-1 \mathrm{a}+1$, sendo igual a zero aceita-se a hipótese nula, $\mathrm{o}$ que significa não representar diferenças entre as amostras (Clarke 1993).

Para a avaliação das relações florísticas e da cobertura entre os períodos e entre as estratificações, foi empregado análise de correspondência retificada - DCA (Hill \& Gauch 1980), utilizando o programa CANOCO para Windows versão 4 (ter Braak \& Smilauer 1998), a partir de uma matriz de cobertura das espécies. Esta análise permite ordenar as linhas de amostragem da vegetação de acordo com a similaridade florística entre elas. Este tipo de análise indireta é indicada para evidenciar padrões de variação na composição de espécies relacionadas com características ambientais (ter Braak \& Smilauer 1998).

Posteriormente realizou-se um Procedimento de Permutação de Resposta Múltipla (MRPP), executada pelo programa PC-ORD 4.0 (McCune \& Mefford 1999), utilizando a distância de Sørensen como medida de dissimilaridade para a abundância de espécies, avaliando se a composição dos grupos formados pelo gradiente observado na DCA difere entre si. Permitindo avaliar se estes são mais similares entre si do que seria esperado caso as amostras estivessem distribuídas ao acaso na área. Onde delta (A) varia de 0 a 1 , tendo o valor máximo quando todas os gradientes apresentam a mesma composição de espécies (McCune \& Mefford 1999).

Os materiais coletados nos inventários foram identificados por meio de comparação com exemplares herborizados depositados no Herbário da Reserva Ecológica do Instituto Brasileiro de Geografia e Estatística (IBGE) e no Herbário da Universidade de Brasília (UB), com auxílio de materiais literários e consulta a especialistas. Os materiais férteis foram herborizados e depositados no Herbário do IBGE. As espécies foram classificadas em famílias de acordo com sistema do Angiosperm Phylogeny Group II (APG II 2003).

\section{Resultados}

Foram amostradas no campo limpo úmido da FAF 98 espécies, distribuídas em 46 gêneros de 24 famílias, sendo 88 espécies, 44 gêneros e 24 famílias no T0 e 67 espécies, 37 gêneros e 19 famílias no T1 (Tab. 1). Dessas 98 espécies registradas, 57 são comuns aos levantamentos, sendo 31 exclusivas do T0 e 10 do T1. A similaridade florística entre os dois inventários foi elevada. O índice de Chao-Sørensen \pm IC foi de 0,84 $\pm 0,074$. Apresentando uma maior similaridade das linhas de amostragens dentro dos anos do que entre eles $(\mathrm{R}=0,58 ; \mathrm{p}<0,001)$

Houve modificação na distribuição das frequências relativas $\left(\chi^{2}=277,25 ; \mathrm{gl}=97 ; \mathrm{p}<0,001\right)$ e também na distribuição das espécies em relação à cobertura relativa $\left(\chi^{2}=156,28 ; \mathrm{gl}=97 ; \mathrm{p}<0,001\right)$, como observado nas dez espécies mais frequentes (Fig. 3) e com maior cobertura (Fig. 4). Entre as espécies perenes comuns nos dois levantamentos $40,48 \%$ aumentaram a cobertura em T1, com destaque para Paspalum lineare e Andropogon virgatus (Fig. 4). A diversidade florística para a comunidade foi elevada, $\mathrm{H}^{\prime}=3,81$ e H' = 3,57, com equitabilidade de 0,85 e 0,85 para T0 e T1, respectivamente. Houve diferença significativa entre as diversidades dos dois períodos $(\mathrm{t}$ $=7,12 ; \mathrm{p}<0,001)$.

Os diagramas resultantes da ordenação por DCA mostraram, de modo geral, que as linhas se distribuem de forma agrupada ao longo dos eixos, indicando relações entre a composição florística e a cobertura (Fig. 5) com o gradiente de umidade e de matéria orgânica no solo identificados em T0, explicados pelo eixo 1, e com menor relação às alterações ao longo do tempo, explicadas pelo eixo 2 . O eixo 1 explicou $12,20 \%$ da variação (autovalor 0,834 ) e o eixo $2,7,50 \%$ (autovalor 0,513, Fig. 5). Houve modificações na cobertura das linhas sazonais (L2, L7 e L13), as quais se tornaram mais semelhantes, em relação à estrutura da composição florística, com as linhas constantemente saturadas por água (L8, L9, L10, L11, L12 e L14) (Fig. 5). A variação na composição e na estrutura na comunidade após sete anos não foi expressiva na DCA, de forma a separar as duas amostragens (T0 e T1). A análise de permutação (MRPP) mostrou que os grupos formados pelo gradiente de umidade e o teor de matéria orgânica observado na DCA são diferentes ( $\mathrm{A}=$ $0,15 ; \mathrm{p}<0,001)$.

\section{Discussão}

A modificação na riqueza de espécies entre T0 e T1 demonstra que a variação da composição florística em comunidades campestres são observadas ao longo dos anos (Dunnett et al. 1998; Anderson \& Inouye 2001; Reis et al. 2006), assim como em um mesmo ano (Cianciaruso \& Batalha 2008; Munhoz \& Felfili 2006b; Munhoz \& Felfili 2008). Alterações essas decorrentes das características específicas das espécies, as quais podem ser influenciadas por fatores climáticos (Guo et al. 2002; Matesanz et al. 2009) e pelo fogo (Luis et al. 2006).

O campo limpo úmido da FAF apresentou uma elevada diversidade, semelhante ao encontrado em áreas úmidas de campo no Distrito Federal (Munhoz \& Felfili 2006b; Munhoz \& Felfili 2008), em Goiás (Cianciaruso \& Batalha 2008) e em São Paulo (Tannus \& Assis 2004). No entanto, 
Tabela 1. Espécies amostradas na comunidade herbácea-arbustiva nos anos de 2000 (T0) (Munhoz 2003) e 2007 (T1), em ordem decrescente de cobertura absoluta (CA) em T0, registradas em uma área de campo limpo úmido da Fazenda Água Fria, Goiás, Brasil. Cobertura relativa (CR); frequência absoluta (FA) e frequência relativa (FR).

\begin{tabular}{|c|c|c|c|c|c|c|c|c|c|}
\hline \multirow{2}{*}{ Espécies } & \multirow{2}{*}{ Famílias } & \multicolumn{4}{|c|}{ T0 } & \multicolumn{4}{|c|}{ T1 } \\
\hline & & $\mathrm{CA}$ & CR & FA & FR & $\mathrm{CA}$ & CR & FA & FR \\
\hline Paspalum lineare Trin. & Poaceae & 44,92 & 14,58 & 32,00 & 4,98 & 78,82 & 33,17 & 58,00 & 11,73 \\
\hline Syngonanthus decorus Moldenke & Eriocaulaceae & 29,16 & 9,46 & 26,00 & 4,05 & 3,72 & 1,57 & 10,00 & 2,02 \\
\hline Rhynchospora emaciata (Nees) Boeck. & Cyperaceae & 16,90 & 5,49 & 12,67 & 1,97 & 0,00 & 0,00 & 0,00 & 0,00 \\
\hline Trachypogon spicatus (L. f.) Kuntze & Poaceae & 15,70 & 5,10 & 20,00 & 3,11 & 9,13 & 3,84 & 11,33 & 2,29 \\
\hline Rhynchospora graminea Uittien & Cyperaceae & 14,99 & 4,87 & 28,00 & 4,36 & 0,00 & 0,00 & 0,00 & 0,00 \\
\hline Lagenocarpus rigidus (Kunth) Nees & Cyperaceae & 12,40 & 4,02 & 20,67 & 3,22 & 11,83 & 4,98 & 20,67 & 4,18 \\
\hline Echinolaena inflexa (Poir.) Chase & Poaceae & 12,08 & 3,92 & 28,00 & 4,36 & 10,05 & 4,23 & 27,33 & 5,53 \\
\hline Froelichiella grisea R.E.Fr. & Amaranthaceae & 10,39 & 3,37 & 35,33 & 5,50 & 2,18 & 0,92 & 17,33 & 3,50 \\
\hline Rhynchospora setacea Vahl & Cyperaceae & 8,88 & 2,88 & 12,00 & 1,87 & 0,62 & 0,26 & 4,00 & 0,81 \\
\hline Xyris tortula Mart. & Xyridaceae & 8,44 & 2,74 & 17,33 & 2,70 & 9,11 & 3,83 & 22,00 & 4,45 \\
\hline Loudetiopsis chrysothrix (Nees) Conert & Poaceae & 7,91 & 2,57 & 9,33 & 1,45 & 8,53 & 3,59 & 10,00 & 2,02 \\
\hline Scleria leptostachya Kunth & Cyperaceae & 7,22 & 2,34 & 12,67 & 1,97 & 2,29 & 0,96 & 5,33 & 1,08 \\
\hline Mesosetum elytrochaetum (Hack.) Swallen & Poaceae & 6,84 & 2,22 & 8,67 & 1,35 & 0,00 & 0,00 & 0,00 & 0,00 \\
\hline Rhynchospora robusta (Kunth) Boeck. & Cyperaceae & 6,80 & 2,21 & 12,67 & 1,97 & 4,40 & 1,85 & 12,67 & 2,56 \\
\hline Panicum cyanescens Nees ex Trin. & Poaceae & 5,68 & 1,84 & 13,33 & 2,07 & 3,45 & 1,45 & 14,67 & 2,96 \\
\hline Syngonanthus gracilis (Bong.) Ruhland & Eriocaulaceae & 5,62 & 1,82 & 16,67 & 2,59 & 0,00 & 0,00 & 0,00 & 0,00 \\
\hline Microlicia viminalis Triana & Melastomataceae & 5,51 & 1,79 & 12,00 & 1,87 & 3,65 & 1,54 & 11,33 & 2,29 \\
\hline Scleria hirtella Sw. & Cyperaceae & 5,22 & 1,69 & 9,33 & 1,45 & 0,00 & 0,00 & 0,00 & 0,00 \\
\hline Vellozia pumila Goethart \& Henrard & Velloziaceae & 4,63 & 1,50 & 7,33 & 1,14 & 3,38 & 1,42 & 6,67 & 1,35 \\
\hline Paspalum scalare Trin. & Poaceae & 4,47 & 1,45 & 6,00 & 0,93 & 4,66 & 1,96 & 6,00 & 1,21 \\
\hline Aristida capillacea Lam. & Poaceae & 4,37 & 1,42 & 10,00 & 1,56 & 0,15 & 0,06 & 1,33 & 0,27 \\
\hline Andropogon virgatus Desv. ex Ham. & Poaceae & 4,18 & 1,36 & 10,67 & 1,66 & 9,84 & 4,14 & 12,67 & 2,56 \\
\hline Xyris ciliata Thunb. & Xyridaceae & 4,12 & 1,34 & 12,00 & 1,87 & 1,75 & 0,74 & 9,33 & 1,89 \\
\hline Vellozia tubiflora (A.Rich.) Kunth & Velloziaceae & 3,92 & 1,27 & 10,67 & 1,66 & 2,99 & 1,26 & 8,67 & 1,75 \\
\hline Exochogyne amazonica C.B. Clarke & Cyperaceae & 3,87 & 1,26 & 13,33 & 2,07 & 2,36 & 0,99 & 11,33 & 2,29 \\
\hline Rhynchospora pilosa (Kunth) Boeck. & Cyperaceae & 3,84 & 1,25 & 7,33 & 1,14 & 0,00 & 0,00 & 0,00 & 0,00 \\
\hline Rhynchospora globosa (Kunth) Roem. \& Schult. & Cyperaceae & 3,82 & 1,24 & 8,67 & 1,35 & 3,28 & 1,38 & 12,67 & 2,56 \\
\hline Paspalum maculosum Trin. & Poaceae & 3,32 & 1,08 & 8,67 & 1,35 & 1,39 & 0,58 & 5,33 & 1,08 \\
\hline Syngonanthus nitens (Bong.) Ruhland & Eriocaulaceae & 3,19 & 1,04 & 12,67 & 1,97 & 6,61 & 2,78 & 26,67 & 5,39 \\
\hline Xyris dawsonii L.B.Sm. \& Downs & Xyridaceae & 2,83 & 0,92 & 9,33 & 1,45 & 0,00 & 0,00 & 0,00 & 0,00 \\
\hline Curtia tenuifolia (Aubl.) Knobl. & Gentianaceae & 2,51 & 0,81 & 12,67 & 1,97 & 0,62 & 0,26 & 6,00 & 1,21 \\
\hline Syngonanthus sp.1 & Eriocaulaceae & 2,31 & 0,75 & 19,33 & 3,01 & 0,00 & 0,00 & 0,00 & 0,00 \\
\hline Bulbostylis sellowiana (Kunth) Palla & Cyperaceae & 2,17 & 0,70 & 7,33 & 1,14 & 0,44 & 0,19 & 4,67 & 0,94 \\
\hline Eugenia cristaensis O. Berg & Myrtaceae & 1,95 & 0,63 & 4,67 & 0,73 & 1,02 & 0,43 & 4,00 & 0,81 \\
\hline Hyptis pycnocephala Benth. & Lamiaceae & 1,93 & 0,63 & 3,33 & 0,52 & 0,00 & 0,00 & 0,00 & 0,00 \\
\hline $\begin{array}{l}\text { Lagenocarpus rigidus subsp tenuifolius (Boeck.) } \\
\text { T. Koyama \& Maguire }\end{array}$ & Cyperaceae & 1,66 & 0,54 & 3,33 & 0,52 & 2,22 & 0,93 & 8,00 & 1,62 \\
\hline
\end{tabular}


Tabela 1. Continuação

\begin{tabular}{|c|c|c|c|c|c|c|c|c|c|}
\hline \multirow{2}{*}{ Espécies } & \multirow{2}{*}{ Famílias } & \multicolumn{4}{|c|}{ T0 } & \multicolumn{4}{|c|}{ T1 } \\
\hline & & $\mathrm{CA}$ & $\mathrm{CR}$ & FA & FR & $\mathrm{CA}$ & $\mathrm{CR}$ & FA & FR \\
\hline Sacciolepis myuros (Lam.) Chase & Poaceae & 1,41 & 0,46 & 4,67 & 0,73 & 0,03 & 0,01 & 1,33 & 0,27 \\
\hline Paepalanthus elongatus Körn. var. niger Moldenke & Eriocaulaceae & 1,38 & 0,45 & 8,00 & 1,24 & 0,18 & 0,08 & 2,00 & 0,40 \\
\hline Paepalanthus elongatus Körn. & Eriocaulaceae & 1,14 & 0,37 & 6,00 & 0,93 & 1,15 & 0,48 & 6,00 & 1,21 \\
\hline Xyris blanchetiana Malme & Xyridaceae & 1,14 & 0,37 & 9,33 & 1,45 & 0,96 & 0,40 & 6,67 & 1,35 \\
\hline Rhynchospora confinis (Nees) C.B.Clarke & Cyperaceae & 1,08 & 0,35 & 4,00 & 0,62 & 0,41 & 0,17 & 2,00 & 0,40 \\
\hline Bulbostylis laeta C.B. Clarke & Cyperaceae & 1,05 & 0,34 & 7,33 & 1,14 & 0,00 & 0,00 & 0,00 & 0,00 \\
\hline Xyris sp.1 & Xyridaceae & 1,05 & 0,34 & 4,67 & 0,73 & 0,00 & 0,00 & 0,00 & 0,00 \\
\hline Mesosetum loliiforme (Hochst. ex Steud.) Chase & Poaceae & 0,94 & 0,31 & 3,33 & 0,52 & 0,52 & 0,22 & 3,33 & 0,67 \\
\hline Xyris diaphanobracteata Kral \& Wand. & Xyridaceae & 0,82 & 0,27 & 4,00 & 0,62 & 1,21 & 0,51 & 5,33 & 1,08 \\
\hline Sisyrinchium vaginatum Spreng. & Iridaceae & 0,78 & 0,25 & 10,67 & 1,66 & 0,43 & 0,18 & 8,00 & 1,62 \\
\hline Irlbachia caerulescens (Aubl.) Griseb. & Gentianaceae & 0,71 & 0,23 & 0,67 & 0,10 & 0,00 & 0,00 & 0,00 & 0,00 \\
\hline Lessingianthus cristalinae (H. Rob.) H. Rob. & Asteraceae & 0,70 & 0,23 & 2,67 & 0,41 & 2,01 & 0,85 & 13,33 & 2,70 \\
\hline Scleria setacea Poir. & Cyperaceae & 0,68 & 0,22 & 2,67 & 0,41 & 0,00 & 0,00 & 0,00 & 0,00 \\
\hline Burmannia flava Mart. & Burmanniaceae & 0,61 & 0,20 & 4,00 & 0,62 & 0,43 & 0,18 & 2,00 & 0,40 \\
\hline Paepalanthus bifidus (Schrader) Kunth & Eriocaulaceae & 0,59 & 0,19 & 4,67 & 0,73 & 0,00 & 0,00 & 0,00 & 0,00 \\
\hline Microlicia ramosa Pilg. & Melastomataceae & 0,55 & 0,18 & 4,00 & 0,62 & 0,22 & 0,09 & 1,33 & 0,27 \\
\hline Calea gardneriana Baker & Asteraceae & 0,54 & 0,18 & 2,67 & 0,41 & 0,96 & 0,40 & 4,67 & 0,94 \\
\hline Epidendrum secundum Jacq. & Orchidaceae & 0,53 & 0,17 & 4,00 & 0,62 & 0,00 & 0,00 & 0,00 & 0,00 \\
\hline Andropogon selloanus (Hack.) Hack. & Poaceae & 0,48 & 0,16 & 4,00 & 0,62 & 0,00 & 0,00 & 0,00 & 0,00 \\
\hline Paepalanthus phaeocephalus Ruhland & Eriocaulaceae & 0,42 & 0,14 & 2,00 & 0,31 & 0,00 & 0,00 & 0,00 & 0,00 \\
\hline Axonopus aureus P. Beauv. & Poaceae & 0,41 & 0,13 & 3,33 & 0,52 & 0,76 & 0,32 & 4,00 & 0,81 \\
\hline Lessingianthus eitenii (H. Rob.) H. Rob. & Asteraceae & 0,35 & 0,11 & 4,00 & 0,62 & 0,11 & 0,05 & 0,67 & 0,13 \\
\hline Mandevilla myriophyllum (Taub.) Woodson & Apocynaceae & 0,34 & 0,11 & 2,67 & 0,41 & 0,69 & 0,29 & 1,33 & 0,27 \\
\hline Cyperus haspan $\mathrm{L}$. & Cyperaceae & 0,32 & 0,10 & 2,00 & 0,31 & 0,20 & 0,08 & 0,67 & 0,13 \\
\hline Xyris paradisiaca Wand. & Xyridaceae & 0,30 & 0,10 & 3,33 & 0,52 & 3,68 & 1,55 & 4,67 & 0,94 \\
\hline Microlicia psammophila Wurdack & Melastomataceae & 0,28 & 0,09 & 1,33 & 0,21 & 0,08 & 0,03 & 0,67 & 0,13 \\
\hline Xyris filifolia A. Nilsson & Xyridaceae & 0,26 & 0,08 & 2,67 & 0,41 & 0,14 & 0,06 & 2,00 & 0,40 \\
\hline Xyris hymenachne Mart. & Xyridaceae & 0,22 & 0,07 & 2,00 & 0,31 & 0,26 & 0,11 & 2,00 & 0,40 \\
\hline Ludwigia nervosa (Poir.) H. Hara & Onagraceae & 0,21 & 0,07 & 1,33 & 0,21 & 0,44 & 0,19 & 4,00 & 0,81 \\
\hline Syngonanthus xeranthemoides (Bong.) Ruhland & Eriocaulaceae & 0,21 & 0,07 & 2,00 & 0,31 & 1,15 & 0,48 & 8,00 & 1,62 \\
\hline Xyris veruina Malme & Xyridaceae & 0,21 & 0,07 & 0,67 & 0,10 & 0,00 & 0,00 & 0,00 & 0,00 \\
\hline Evolvulus lagopodioides Meisn. & Convolvulaceae & 0,18 & 0,06 & 2,00 & 0,31 & 0,30 & 0,13 & 2,00 & 0,40 \\
\hline Lessingianthus linearifolius (Less.) H. Rob. & Asteraceae & 0,18 & 0,06 & 1,33 & 0,21 & 0,12 & 0,05 & 1,33 & 0,27 \\
\hline Utricularia amethystina Salzm. ex A. St.-Hil. \& Girard & Lentibulariaceae & 0,18 & 0,06 & 2,67 & 0,41 & 0,04 & 0,02 & 0,67 & 0,13 \\
\hline Paspalum minarum Hack. & Poaceae & 0,17 & 0,06 & 1,33 & 0,21 & 0,34 & 0,14 & 1,33 & 0,27 \\
\hline Xyris savanensis Miq. & Xyridaceae & 0,14 & 0,05 & 2,00 & 0,31 & 0,00 & 0,00 & 0,00 & 0,00 \\
\hline Turnera trigona Urb. & Turneraceae & 0,13 & 0,04 & 2,00 & 0,31 & 0,00 & 0,00 & 0,00 & 0,00 \\
\hline
\end{tabular}


Tabela 1. Continuação

\begin{tabular}{|c|c|c|c|c|c|c|c|c|c|}
\hline \multirow{2}{*}{ Espécies } & \multirow{2}{*}{ Famílias } & \multicolumn{4}{|c|}{ T0 } & \multicolumn{4}{|c|}{$\mathrm{T} 1$} \\
\hline & & $\mathrm{CA}$ & $\mathrm{CR}$ & FA & FR & $\mathrm{CA}$ & $\mathrm{CR}$ & $\mathrm{FA}$ & FR \\
\hline Drosera montana A. St.-Hil. & Droseraceae & 0,12 & 0,04 & 4,00 & 0,62 & 0,00 & 0,00 & 0,00 & 0,00 \\
\hline Trimezia sp.1 & Iridaceae & 0,12 & 0,04 & 1,33 & 0,21 & 0,00 & 0,00 & 0,00 & 0,00 \\
\hline Paepalanthus acanthophyllus Ruhland & Eriocaulaceae & 0,10 & 0,03 & 0,67 & 0,10 & 0,00 & 0,00 & 0,00 & 0,00 \\
\hline Xyris tenella Kunth & Xyridaceae & 0,10 & 0,03 & 1,33 & 0,21 & 0,00 & 0,00 & 0,00 & 0,00 \\
\hline Cyperus schoenomorphus Steud. & Cyperaceae & 0,08 & 0,03 & 0,67 & 0,10 & 0,00 & 0,00 & 0,00 & 0,00 \\
\hline Mimosa setosa Benth. & Fabaceae & 0,08 & 0,03 & 0,67 & 0,10 & 0,04 & 0,02 & 0,67 & 0,13 \\
\hline Borreria irwiniana E.L. Cabral & Rubiacea & 0,07 & 0,02 & 1,33 & 0,21 & 0,00 & 0,00 & 0,00 & 0,00 \\
\hline Hyptis cruciformis Epling & Lamiaceae & 0,07 & 0,02 & 0,67 & 0,10 & 0,09 & 0,04 & 0,67 & 0,13 \\
\hline Utricularia adpressa Salzm. ex A. St.-Hil. \& Girard & Lentibulariaceae & 0,07 & 0,02 & 2,67 & 0,41 & 0,00 & 0,00 & 0,00 & 0,00 \\
\hline Rhynchospora marisculus Lindl. ex Nees & Cyperaceae & 0,06 & 0,02 & 0,67 & 0,10 & 0,52 & 0,22 & 2,67 & 0,54 \\
\hline Bulbostylis jacobinae Lindm. & Cyperaceae & 0,05 & 0,02 & 0,67 & 0,10 & 0,00 & 0,00 & 0,00 & 0,00 \\
\hline Ipomoea pinifolia Meisn. & Convolvulaceae & 0,04 & 0,01 & 1,33 & 0,21 & 0,00 & 0,00 & 0,00 & 0,00 \\
\hline Paepalanthus eriocauloides Ruhland & Eriocaulaceae & 0,04 & 0,01 & 1,33 & 0,21 & 0,22 & 0,09 & 1,33 & 0,27 \\
\hline Habenaria magniscutata Catling & Orchidaceae & 0,01 & 0,00 & 0,67 & 0,10 & 0,00 & 0,00 & 0,00 & 0,00 \\
\hline Polygala carphoides Chodat & Polygalaceae & 0,01 & 0,00 & 0,67 & 0,10 & 0,00 & 0,00 & 0,00 & 0,00 \\
\hline Cipura paludosa Aubl. & Iridaceae & 0,00 & 0,00 & 0,00 & 0,00 & 0,06 & 0,03 & 0,67 & 0,13 \\
\hline Hyptis selaginifolia Mart. ex Benth. & Lamiaceae & 0,00 & 0,00 & 0,00 & 0,00 & 2,00 & 0,84 & 3,33 & 0,67 \\
\hline Microlicia castrata Naudin & Melastomataceae & 0,00 & 0,00 & 0,00 & 0,00 & 0,33 & 0,14 & 2,00 & 0,40 \\
\hline Microstachys bidentata (Mart. \& Zucc.) Ess & Euphorbiaceae & 0,00 & 0,00 & 0,00 & 0,00 & 0,03 & 0,01 & 0,67 & 0,13 \\
\hline Paepalanthus canescens Körn. & Eriocaulaceae & 0,00 & 0,00 & 0,00 & 0,00 & 8,39 & 3,53 & 13,33 & 2,70 \\
\hline Riencourtia oblongifolia Gardner & Asteraceae & 0,00 & 0,00 & 0,00 & 0,00 & 0,05 & 0,02 & 2,00 & 0,40 \\
\hline Scleria reticularis Michx. & Cyperaceae & 0,00 & 0,00 & 0,00 & 0,00 & 9,46 & 3,98 & 14,67 & 2,96 \\
\hline Syngonanthus sp. 2 & Eriocaulaceae & 0,00 & 0,00 & 0,00 & 0,00 & 2,69 & 1,13 & 7,33 & 1,48 \\
\hline Trachypogon macroglossus Trin. & Poaceae & 0,00 & 0,00 & 0,00 & 0,00 & 4,35 & 1,83 & 7,33 & 1,48 \\
\hline Xyris sp.2 & Xyridaceae & 0,00 & 0,00 & 0,00 & 0,00 & 0,05 & 0,02 & 0,67 & 0,13 \\
\hline
\end{tabular}

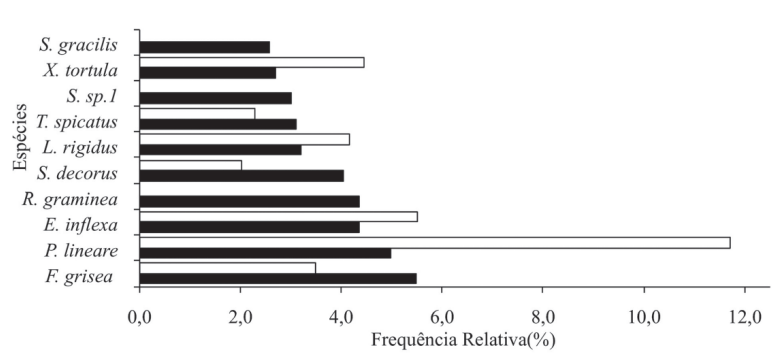

Figura 3. As dez espécies com maior frequência relativa, nos períodos de amostragem, para a flora herbáceo-arbustiva de uma área de campo limpo úmido na Fazenda Água Fria, Goiás, Brasil. (Nomes das espécies, ver Tab. 1). $\mathbf{\square}$ Frequência Relativa em T0 (2000); $\square$ Frequência Relativa em T1 (2007).

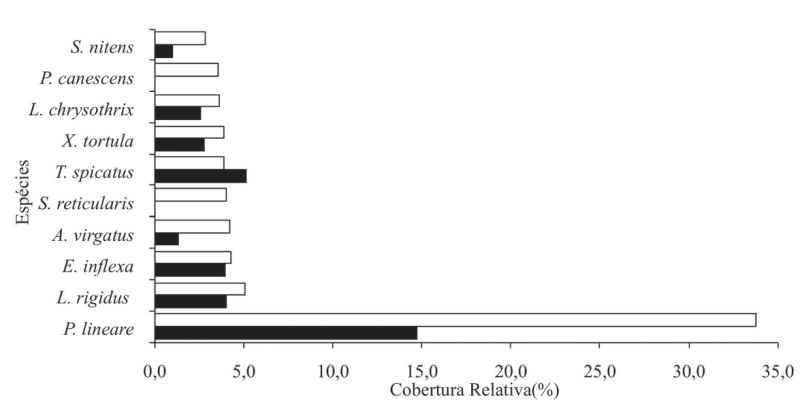

Figura 4. As dez espécies com maior cobertura relativa nos períodos de amostragem, para a flora herbáceo-arbustiva de uma área de campo limpo úmido na Fazenda Água Fria, Goiás, Brasil. (Nomes das espécies, ver Tabela 1). Cobertura Relativa em T0; $\square$ Cobertura Relativa em T1. 


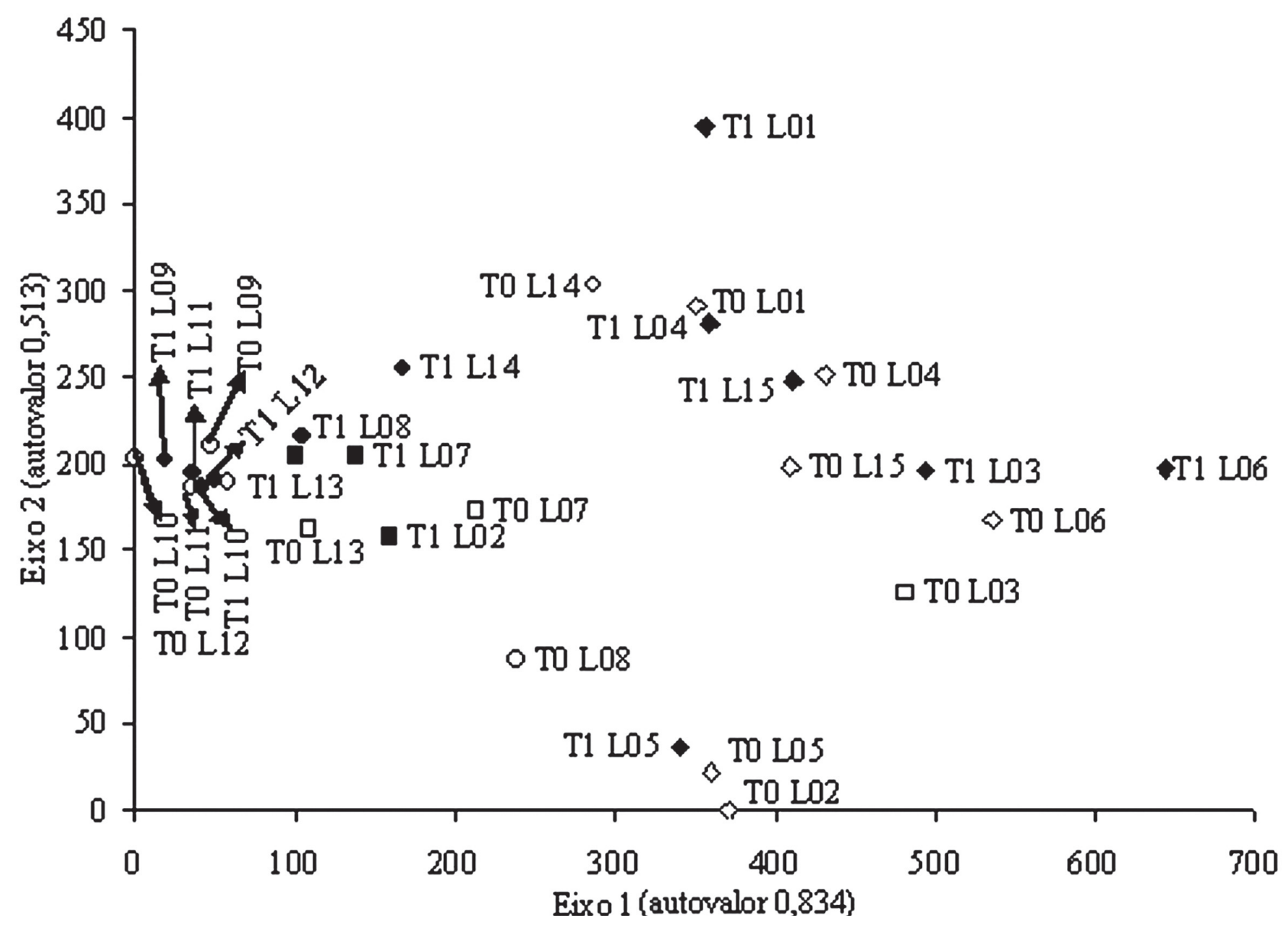

Figura 5. Ordenação das 15 linhas amostradas em uma área de campo limpo úmido da Fazenda Água Fria, Goiás, Brasil, a partir de uma Análise de Correspondência Retificada (DCA) da matriz de cobertura. L $(\mathrm{n})$ = número da linha, T0 = primeiro inventário (2000, símbolos preenchidos) e T1= segundo inventário (2007, símbolos vazios). Segundo Munhoz (2003): $\bigcirc=$ linhas em solos permanentemente saturados por água e alto teor de matéria orgânica; $\square=$ linhas em solos inundados somente no auge da estação chuvosa e com elevado teor de matéria orgânica; $\diamond$ = linhas em solos com menores porcentagens de umidade gravimétrica e as men ores taxas de matéria orgânica.

a diferença significativa entre as diversidades, entre os tempos de amostragem na FAF, foi devido à ausência de 31 espécies, que representavam $21,84 \%$ da cobertura relativa da comunidade no $\mathrm{T} 0 \mathrm{e}$ ao aumento da cobertura em $41,72 \%$ de 21 espécies em sete anos, sendo que somente Paspalum lineare Trin. aumentou sua cobertura relativa em $18,59 \%$.

O resultado expressivo de Paspalum lineare, provavelmente, deve-se ao seu hábito cespitoso, entouceirado e por se tratar de uma espécie perene. As pesquisas mostram que as espécies respondem de forma diferente a proteção contra os distúrbios dependendo de sua arquitetura e tamanho (Canales \& Silva 1987). A ausência de distúrbios ao longo dos anos e o equilíbrio da interação da precipitação com a temperatura entre os anos, pode também favorecer o aumento da cobertura de espécies perenes e o sucesso das espécies anuais está relacionado à importância dos balanços hídricos durante o ano (Matesanz et al. 2009). Desta forma, a elevação da precipitação em Alto Paraíso de Goiás pode ter levado a um aumento das coberturas das espécies anuais Mandevilla myriophyllum e Paepalanthus eriocauloides, que ocorreram em T0 somente no período das chuvas ou em linhas saturadas durante todo ano (Munhoz 2003). Porém, a ausência de algumas espécies em $\mathrm{T} 1$ pode ter ocorrido pela expansão das espécies perenes dominantes. Experimentos com o controle de precipitação em campos na Inglaterra mostraram que a elevação da umidade pela precipitação favorece o aumento da cobertura de espécies perenes dominantes, evitando o estabelecimento de espécies anuais (Morecroft et al. 2004).

As espécies Rhynchospora emaciata e Rhynchospora graminea, que não ocorreram em $\mathrm{T} 1$, foram consideradas como indicadoras de comunidades sazonais na área por Munhoz (2003), pois foram restritas às linhas que apresentavam flutuação no regime hídrico do solo em T0. A espécie Trachypogon spicatus, que reduziu a cobertura em T1, foi registrada frequentemente em porções mais secas do campo limpo úmido da FAF e de veredas (Araújo et al. 2002), em campo sujo (Munhoz \& Felfili 2006b) e 
também na camada rasteira de áreas de cerrado sensu stricto (Felfili et al. 1994; Silva \& Nogueira 1999). Essas espécies sem registro ou que reduziram a cobertura em $\mathrm{T} 1$ são comumente amostradas em comunidades sazonais à mais secas.

O evento ocorrido pelo aumento no volume das chuvas, causado pelo El Niño moderado e fraco, segundo o Centro de Previsão de Tempo e Estudos Climáticos CPTEC (INPE, 2003), no intervalo entre os inventários, proporcionou à área de estudo um maior volume de água e consequentemente uma menor flutuação do lençol freático, podendo ter desfavorecido o aumento da cobertura das espécies das zonas mais secas do campo. Anderson \& Inouye (2001), avaliando mudanças na abundância e diversidade da vegetação em comunidades campestres nos Estados Unidos durante 45 anos, verificaram um atraso de 2 a 4 anos na resposta da cobertura das principais espécies ou grupos da comunidade em relação a precipitação.

A umidade e os teores de matéria orgânica no solo, que influenciaram na composição, estrutura e distribuição espacial das espécies, no T0 (Munhoz 2003), permaneceram importantes na distribuição das linhas no diagrama da DCA no T1. No entanto, nessa análise as linhas do T1 estão mais agrupadas no centro e em direção ao gradiente de solos permanentemente alagados e com alto teor de matéria orgânica, provavelmente devido a maior umidade no solo proporcionada pela elevação da precipitação. A elevação do lençol freático em campos da Holanda, durante cinco anos, levou a dominância de espécies indicadoras de condições úmidas (Oomes et al. 1996). Lopes \& Schiavini (2007) estudando uma comunidade arbórea de mata de galeria encontraram em um intervalo de 13 anos mudanças na estrutura e na composição florística devido às mudanças nas condições do solo, principalmente a umidade. À medida que os fatores ambientais sofrem mudanças, ocorrem substituições das espécies, com reflexo na cobertura e frequência das mesmas na comunidade. Portanto, o monitoramento da área em longo prazo é necessário para que se confirmem as alterações, assim como a observação da flutuação do lençol freático.

A área campestre estudada parece sofrer mudanças na sua composição florística e estrutural, sendo as variações menores na composição florística do que na estrutura ao longo do tempo, com o aumento da cobertura de espécies perenes, cespitosas e entouceiradas que foram favorecidas pela maior umidade no solo em resposta à elevação da pluviosidade da região. Monitoramentos de longo prazo e em curtos intervalos de tempo em um número maior de comunidades, assim como dos fatores abióticos, devem averiguar as influências de fenômenos climáticos, na dinâmica de comunidades sem distúrbios.

\section{Agradecimentos}

Ao CNPq pelo apoio concedido ao projeto. À equipe técnica do herbário do IBGE, especialmente à Marina Fonseca. Ao grupo da Universidade Católica de Brasília, especialmente a Ana Carolina Gomes Côrrea. Pela identificação do material botânico, agradecemos aos taxonomistas: A. Goldberg (Turneraceae); A. P. Prata (Cyperaceae); C. Fagg (Fabaceae); D.C.Wasshausen (Acanthaceae); E. Guimarães (Gentianaceae); E. L. Cabral (Rubiaceae); G.L. Webster (Euphorbiaceae); H. Robinson (Asteraceae); J.N. Nakajima (Asteraceae); Josafa Carlos de Siqueira (Amaranthaceae); Katia Calago (Asteraceae); Luciano B. Biancheti (Orchidaceae); C. Proença (Myrtaceae e várias famílias); M. Gomes (Rubiaceae); M. Magenta (Asteraceae); M.C. Marques (Polygalaceae); M. G. L.Wanderley (Xyridaceae); N. Hensold (Eriocaulaceae); P.J.M. Maas (Burmaniaceae); L. Milhomem (Asteraceae); Ronaldo Marquete (Eriocaulaceae); R. Marquete (Apocynaceae e Gentianaceae); R.C. Oliveira (Poaceae); S.F. Smith (Xyridaceae); T. Filgueiras (Poaceae) e V. L. Rivera \& L. Fonseca (Asteraceae).

\section{Referências Bibliográficas}

Anderson, J.E. \& Inouye, R.S. 2001. Landscape-scale changes in plant species abundance and biodiversity of a Sagebrush Steppe over 45 years. Ecological Monographs 71: 531-556.

Angiosperm Phylogeny Group II (APG II). 2003. An update of the angiosperm phylogeny group classification for the orders and families of flowering plants: APGII. Botanical Journal of the Linnean Society 141(4):399-436.

Araújo, G.M.; Barbosa, A.A.A.; Arantes, A.A. \& Alice, F.A. 2002. Composição florística de veredas no Município de Uberlândia, MG. Revista Brasileira de Botânica 25: 475-493.

Bakker, J.P; Olff, H.; Willems, J.H. \& Zobel, M. 1996. Why do we need permanent plots in the study of long-term vegetation dynamics? Journal of Vegetation Science 7: 147-156.

Canales, J. \& Silva, J.F. 1987. Efecto de uma quema sobre el crescimiento y demografia de vastagos em Sporobulus cubensis. Acta Oecologica 8: 391-401.

Canfield, R. 1941. Application of line interception in sampling range vegetation. Journal of Forestry 39: 388-394.

Canfield, R. 1950. Sampling range by the line interception method. Southwestern Forest and Range Experiment Station.

Chao, A.; Chazdon, R.L., Colwell; R.K. \& Shen, T.J. 2005. A new statistical approach for assessing similarity of species composition with incidence and abundance data. Ecology Letters 8: 148-159.

Chao, A.; Chazdon, R.L.; Colwell; R.K. \& Shen, T.J. 2006. Abundance-based similarity indices and their estimation when there are unseen species in samples. Biometrics 62: 361-371.

Chapin, F.S.; Shaver, G.R.; Giblin, A.E.; Nadelhoffer, K.J. \& Laundre, J.A. 1995. Responses of arctic tundra to experimental and observed changes in climate. Ecology 76: 694-711.

Chase, J.M. 2003. Community assembly: why should history matter? Oecologia 136: 489-498.

Cianciaruso, M.V. \& Batalha, M.A. 2008. A year in a Cerrado wet grassland: a non-seasonal island in a seasonal savanna environment. Brazilian Journal of Biology 68: 1519-6984.

Clarke, K.R. 1993. Non-parametric multivariate analysis of changes in community structure. Australian Journal of Ecology 18: 117-143.

Colwell, R.K. 2006. Estimates: Statistical Estimation of Species Richness and Shared Species from Samples, Version 8.0. User's Guide and application published at: http://viceroy.eeb.uconn.edu/ estimates. Persistent URL http://purl.oclc.org/estimates. 
Dunnett, N.P.; Willis, A.J.; Hunt, R. \& Grime, J.P. 1998. A 38-years study of relations between weather and vegetation dynamics in road verges near Bibury, Gloucestershire. Journal of Ecology 86: 610-623.

Felfili, J.M. 2007. A Chapada dos Veadeiros. Pp. 17-23. In: Felfili, J.M.; Rezende, A.V.; da Silva-Júnior, M.C. (Org.). Biogeografia do Bioma Cerrado - Vegetação e solos da chapada dos Veadeiros. Brasilia, Editora Universidade de Brasília.

Felfili, J.M.; Filgueiras, T.S.; Haridasan, M.; Silva-Júnior, M.C., Mendonça, R.C. \& Resende, A.V. 1994. Projeto Biogeografia do Bioma Cerrado: Vegetação \& Solos. Caderno de Geociências 12: 75-166.

Filgueiras, T.S. 2002. Herbaceous plant communities. Pp.121-139. In: Oliveira, P.S. \& Marquis, J.R. (Eds.) The Cerrados of Brazil: Ecology and natural history of a neotropical savanna. New York, Columbia University Press.

Fukami, T.; Bezemer, T.M.; Mortimer, S.R. \& van der Putten, W.H. 2005. Species divergence and trait convergence in experimental plant community assembly. Ecology Letters 8: 1283-1290.

Guo, Q.; Brown, J.H. \& Valone, T.J. 2002. Long-term dynamics of winter and summer annual communities in the Chihuahuan Desert. Journal of Vegetation Science 13: 575-584.

Harley, R.M. 1995. Introdução. Pp. 43-78. In: Stannard, B.L. (Ed.) Flora of the Pico das Almas, Chapada Diamantina, Bahia, Brazil. Kew, Royal Botanic Gardens.

Herben, T.; During, H.J. \& Law, R. 2000. Spatio-Temporal Patterns in Grassland Communities. Pp. 48-64. In: Dieckmann, U. Law, R. \& Metz, J.A. (Eds) The Geometry of Ecological Interactions: Simolifying Spatial Complexity. Cambridge, Cambridge University Press.

Hill, M.O. \& Gauch, H.G. 1980. Detrended Correspondence Analysis, an improved ordination technique. Vegetatio 42: 47-58.

INPE, 2003. Climatologias de precipitação e temperatura. Instituto Nacional de Pesquisas Espaciais. http://www. Cptec.inpe.br/clima/ monit/monitor_brasil.shtml (Acesso em 10/03/2010).

Junk, W.J. 2003. Long-term environmental trends and the future of tropical wetlands. Environmental Conservation 29: 414-435.

Kent, M. \& Coker, P. 1992. Vegetation description and analysis; a pratical approach. London, Belhaven Press.

Köppen, W. 1948. Climatologia: com um Estudio de los Climas de la Tierra. Trad. P.R. Hendrichs Pérez. Mexico, Fondo de Cultura Economica.

Luis, M. de; Raventós, J. \& González-Hidalgo, J.C. 2006. Post-fire vegetation sucesión in Mediterranean gorse shrublands. Acta Oecologica 30: 54-61.

Lopes, S.F, \& Schiavini, I. 2007. Dinâmica da comunidade arbórea de Mata de Galeria da Estação Ecológica do Panga, Minas Gerais, Brasil. Acta Botanica Brasilica 21: 249-261.

Matesanz, S.; Brooker, R.W.; Valladares, F. \& Klotz, S. 2009. Temporal dynamics of marginal steppic vegetation over a 26-year period of substantial environmental change. Journal of Vegetation Science 20: 299-310.

McCune, B. \& Mefford, M.J. 1999. PC-ORD. Multivariate analysis of ecological data, Version 4.19. Oregon, MjM Software Design.

Morecroft, M.D.; Taylor, M.E. \& Whitehouse, A.T. 2004. Changing precipitation patterns alter plant community dynamics and succession in an ex-arable grassland. Functional Ecology 18: 648- 655.

Munhoz, C.B.R. 2003. Padrões de distribuição sazonal e espacial das espécies do estrato herbáceo-subarbustivo em comunidades de campo limpo úmido e de campo sujo. Tese de doutorado. Brasília, Universidade de Brasília.

Munhoz, C.B.R. \& Felfili, J.M. 2006a. Floristics of the herbaceous and subshrub layer of a moist grassland in the Cerrado Biosphere Reserve (Alto Paraíso de Goiás), Brasil. Edinburgh Journal of Botany 63: 343-354.
Munhoz, C.B.R. \& Felfili, J.M. 2006b. Fitossociologia do estrato herbáceosubarbustivo de uma área de campo sujo no Distrito Federal, Brasil. Acta Botanica Brasilica 20: 671-685.

Munhoz, C.B.R. \& Felfili, J.M. 2007. Florística do estrato herbáceosubarbustivo de um campo limpo úmido em Brasília, Brasil. Biota Neotropica 7: 205-215.

Munhoz, C.B.R. \& Felfili, J.M. 2008. Fitossociologia do estrato herbáceosubarbustivo em campo limpo úmido no Distrito Federal, Brasil. Acta Botanica Brasilica 22: 905-913.

Munhoz, C.B.R.; Felfili, J.M. \& Rodrigues, C. 2008. Species-environment relationship in the herb-subshrub layer of a moist Savanna site, Federal District, Brazil. Brazilian Journal of Biology 68(1): 25-35.

Munhoz, C.B.R. \& Proença, C. 1998. Composição florística no município de Alto Paraíso de Goiás na Chapada dos Veadeiros. Boletim do Herbário Ezechias Paulo Heringer 3: 102-150.

Oomes, M.J.M.; Olff, H. \& Altena, H.J. 1996. Effects of vegetation management and raising the water table on nutrient dynamics and vegetation change in a wet grassland. J. Appl. Ec Journal of Applied Ecology 33: 576-588.

Parmesan, C. \& Yohe, G. 2003. A globally coherent fingerprint of climate change impacts across natural systems. Nature 421: 37-42.

R Development Core Team. 2006. R: A language and environment for statistical computing. R Foundation for Statistical Computing, Vienna, Disponível em: http://www.R-project.org.

Rees, M.; Condit, R.; Crawley, M.; Pacala, S. \& Tilman, D. 2001. Long-Term Studies of Vegetation Dynamics. Science 293: 650-655.

Reis, A.M.S.; Araujo, E.L.; Ferraz, E.M.N. \& Moura, A.N. 2006. Inter-annual variations in the floristic and population structure of an herbaceous community of "caatinga" vegetation in Pernambuco, Brazil. Revista Brasileira de Botânica 29: 497-508.

Ribeiro, J.F. \& Walter, B.M.T. 2008. As principais fitofisionomias do bioma Cerrado. Pp. 151-199. In: S. M. Sano; S.P. Almeida \& J.F. Ribeiro (Eds.). Cerrado: Ecologia e flora. Brasília, EMBRAPA-CPAC.

Sarmiento, G. 1983. The savannas of Tropical America. Pp. 245-288. In: Bourliere, F. (Ed.). Ecossystems of the world: Tropical Savannas. Amsterdam, Elsevier.

Sarmiento, G.; Pinillos, M.; Silva, M.P. \& Acevedo, D. 2004. Effects of soil water regime and grazing on vegetation diversity and production in a hyperseasonal savanna in the Apure Llanos, Venezuela. Journal of Tropical Ecology 20(2): 209-220.

Simon, M.F. \& Proença, C. 2000. Phytogeographic patterns of Mimosa (Mimosoideae, Leguminosae) in the Cerrado biome of Brazil: an indicator genus of highaltitude centers of endemism? Biological Conservation 96: 279-296.

Silva, M.A. \& Nogueira, P.E. 1999. Avaliação fitossociológica do estrato arbustivoherbáceo em cerrado stricto sensu após incêndio acidental, no Distrito Federal, Brasil. Boletim do Herbário Ezechias Paulo Heringer 4: 65-78.

Sistema Nacional de Informações sobre Recursos Hídricos - SNIRH; Operadora da Estação: Furnas Centrais Elétricas S.A. http://www. ana.gov.br/portalsnirh. (Acesso em 25/02/ 2010)

Tannus, J.L.S. \& Assis, M.A. 2004. Composição de espécies vasculares de campo sujo e campo úmido em área de Cerrado, Itirapina - SP, Brasil. Revista Brasileira de Botânica 27: 489-506.

ter Braak, C.J.F. \& Smilauer, P. 1998. CANOCO Reference manual and user's guide to Canoco for Windows: software for canonical community ordination (version 4). New York, Microcomputer Power, Ithaca.

Weiher, E. \& Keddy, P.A. 1995. Assembly rules, null models, and trait dispersion: new questions from old patterns. Oikos 74: 159-164.

Zar, J. H. 1999. Biostatical analysis. New Jersey, Prentice-Hall. 